\title{
DHEA promotes osteoblast differentiation by regulating the expression of osteoblast-related genes and $\mathrm{Foxp}^{+}$regulatory $\mathrm{T}$ cells
}

\author{
Xuemin Qiu ${ }^{1,2}$, Yuyan Gui ${ }^{1,2}$, Yingping Xu ${ }^{1,2}$, Dajin $\mathrm{Li}^{1, *}$, Ling Wang ${ }^{1,2, *}$ \\ ${ }^{1}$ Obstetrics and Gynecology Hospital, Fudan University, Shanghai, China; \\ ${ }^{2}$ Shanghai Key Laboratory of Female Reproductive and Endocrine-related Disorders, Shanghai, China.
}

\begin{abstract}
Summary
Several studies have reported that dehydroepiandrosterone (DHEA) promotes osteoblast proliferation and inhibits osteoblast apoptosis and that DHEA inhibits osteoclast maturation. However, whether DHEA regulates osteoblast differentiation remains unclear. The present study first examined the effect of DHEA on bone morphology in vivo. DHEA was found to increase bone volume (BV), bone mineral density (BMD), and the number of trabeculae in bone (Th.N) and it was found to decrease trabecular spacing in bone (Th.sp) in ovariectomized (OVX) mice. Next, the effect of DHEA on osteoblast differentiation was examined in vitro and osteoblastogenesis-related marker genes, such as Runx2, Osterix, Collagen1, and Osteocalcin, were also detected. DHEA increased osteoblast production in mesenchymal stem cells (MSCs) cultured in osteoblastogenic medium, and DHEA increased the expression of Runx2 and osterix, thereby increasing the expression of osteocalcin and collagen1. Immune cells and bone interact, so changes in immune cells were detected in vivo. DHEA increased the number of Foxp3 $3^{+}$regulatory $\mathrm{T}$ cells (Tregs) in the spleen but it did not affect CTLA-4 or IL-10. When MSCs were treated with DHEA in the presence of Tregs, alkaline phosphatase (ALP) activity increased. Osteoblasts and adipocytes are both generated by MSCs. If osteoblast differentiation increases, adipocyte differentiation will decrease, and the reverse also holds true. DHEA was found to increase the number of adipocytes in osteoblastogenic medium but it had no effect on the number of adipocytes and expression of PPAR $\gamma$ mRNA in adipogenic medium. This finding suggests that osteoblasts may be involved in adipocyte production. In conclusion, the current results suggest that DHEA can improve postmenopausal osteoporosis (PMO) by up-regulating osteoblast differentiation via the up-regulation of the expression of osteoblastogenesis-related genes and via an increase in Foxp3 ${ }^{+}$Tregs.
\end{abstract}

Keywords: DHEA, postmenopausal osteoporosis, MSCs, osteoblast, adipocyte, regulatory T cells

\section{Introduction}

The level of dehydroepiandrosterone (DHEA) in postmenopausal women is lower than that in fertile women, which suggests the potential clinical benefit of

\section{*Address correspondence to:}

Dr. Dajin Li, Laboratory for Reproductive Immunology, Hospital and Institute of Obstetrics and Gynecology, Fudan University Shanghai Medical College, No.413, Zhaozhou Road, Shanghai 200011, China

E-mail:djli@shmu.edu.cn

Dr. Ling Wang, Obstetrics \& Gynecology Hospital, Fudan University Shanghai Medical College, 413 Zhaozhou Road, Shanghai 200011, China.

E-mail: dr.wangling@vip.163.com
DHEA as treatment for postmenopausal osteoporosis (PMO) (1). Treatment of postmenopausal osteoporosis is mainly hormone replacement therapy, but this therapy is often accompanied by adverse reactions, limiting its use $(2,3)$. The pressing task is to identify a new way to treat PMO that can prevent osteoporosis without causing adverse reactions.

Mesenchymal stem cells (MSCs) are able to differentiate into multiple types of cells. Under certain conditions, MSCs can be induced to differentiate into osteoblasts, adipocytes, cartilage cells, and other types of cells $(4,5)$. Inducing MSCs to differentiate into osteoblasts and inhibiting MSCs from differentiating into adipocytes is crucial to preventing PMO.

DHEA begins to increase when the adrenal cortex 
begins to function and it gradually decreases after puberty. Along with other endocrine hormones, DHEA in the blood decreases with the onset of menopause. DHEA can prevent diabetes, cancer, and heart disease and it can enhance immune function, suggesting that DHEA can prolong survival and improve the quality of life.

DHEA has a positive effect on bone metabolism (6$8)$. DHEA can promote the growth and proliferation of osteoblasts, and mitogen-activated protein kinase signaling pathways are required for expression of osteoblast-specific genes. DHEA can promote a shift in the Th1/Th2 ratio towards Th2, thus improving immunity in a model of PMO.

Immune cells and bone interact (9-11). Regulatory $\mathrm{T}$ cells (Tregs) are important immunosuppressive cells that regulate the functioning of osteoblasts and osteoclasts $(12,13)$. Whether DHEA affects immune cells and then affects bone metabolism is unclear. Whether DHEA affects the differentiation of MSCs into osteoblasts or adipocytes and the means by which it does so are still unknown. The present study will explore the mechanisms by which DHEA affects the differentiation of MSCs.

\section{Materials and Methods}

\subsection{Mice and reagents}

C57BL/6 mice (6-8 weeks of age) were provided by the Laboratory Animal Facility of the Chinese Academy of Sciences (Shanghai, China) and were housed and handled in accordance with the guidelines of the Chinese Council for Animal Care. Sham-operated mice were used as controls, and ovariectomized (OVX) mice were used as a model of PMO. Mice were divided into 3 groups and then treated with different drugs, i.e. estrogen, DHEA, or saline. MEM, estrogen, DHEA, PBS and saline, and ALP solution/alizarin red solution were purchased from Sigma-Aldrich Co (Saint Louis, MO, USA). Flow cytometry antibodies CD4-FITC, CD25-APC, CTLA-4-PE, IL-10-PE, Foxp3-PE were purchased from eBioscience (San Diego, CA, USA).

\subsection{Experimental protocols in vivo}

Animals were anesthetized with $10 \%$ chloral hydrate. Mice in the sham-operated group underwent surgery but were not OVX. Mice in the treatment groups underwent a bilateral ovariectomy and were divided into 3 groups (OVX, OVX + DHEA, and OVX + E2). One week later, the sham-operated group was treated with saline (containing $0.1 \%$ ethanol), and OVX mice group in the treatment groups were given saline, DHEA $(5 \mathrm{mg}$ / $\mathrm{kg}$ per day orally), or estrogen $(100 \mu \mathrm{g} / \mathrm{kg}$ per day orally) (6). The four groups received equal quantities of fluids at the same time during treatment. All mice were sacrificed after 3 months of treatment. Blood was collected from the heart and serum was stored at $-80^{\circ} \mathrm{C}$ for use in cell culture, the spleen was collected for analysis of Tregs, and femurs were stored for micro-CT analysis (14).

\subsection{Flow cytometry (FCM)}

The spleen was harvested from mice in every group and mechanically disrupted in $10 \mathrm{~mL}$ of PBS. The cell suspension was then filtered through nylon mesh with a pore size of $110 \mu \mathrm{m}$ and treated with $\mathrm{NH} 4 \mathrm{Cl} /$ Tris buffer to remove RBC. The cells were then washed three times and transferred into tubes $(100 \mu \mathrm{L}$ per tube) for immunolabeling. The cells were fixed, permeabilized, and stained for Foxp3, IL-10, CTLA4 using PE-labeled antibodies after cells were labeled with CD4 (FITC) and CD25 (APC). The cells were then washed twice and resuspended in PBS for FCM with a flow cytometer (Becton Dickinson, Palo Alto, CA, USA). PE-conjugated isotypes were used as controls. Statistical analysis was done using isotype-matched controls.

\subsection{MSC culture}

MSCs were cultured as previously described (15). Mice were anesthetized with $10 \%$ chloral hydrate and immersed in $75 \%$ ethanol for $10 \mathrm{~min}$. Under aseptic conditions, the femur was removed and rinsed three times with PBS. The epiphyseal end of the femur was removed, revealing the marrow cavity. Bone marrow was removed, placed in L-DMEM with penicillin and streptomycin, and repeatedly sieved to obtain a single cell suspension. The suspension was centrifuged at 1,000 r/min for $5 \mathrm{~min}$ and the supernatant was discarded. The concentrated cells were placed in culture bottles at a concentration of $1 \times 10^{9} \mathrm{~L}^{-1}$ cells. Cells were cultured at $37^{\circ} \mathrm{C}$ in a $5 \% \mathrm{CO}_{2}$ environment with saturated humidity.

Medium was supplemented after $48 \mathrm{~h}$. Medium was completely replaced with fresh medium every $3 \mathrm{~d}$. Cells covering the bottom of the culture bottle formed a 70 $80 \%$ confluent monolayer. After digestion with $0.25 \%$ trypsin, cells were subcultured at a ratio of $1: 2$.

\subsection{MSC-derived osteoblasts}

MSCs were seeded as previously reported (16) in order to induce differentiation into osteoblasts. Twenty-four hours after seeding, the growth medium was replaced with osteoblastogenic medium (OM) and 10\% FCS was replaced with solvent alone (control), $10^{-7} \mathrm{M}$ DHEA, or $10^{-9} \mathrm{M}$ E2. Medium was changed every 2-3 days. After 14-21 days of osteogenic differentiation, cells were fixed with $70 \%$ ethanol for $1 \mathrm{~h}$, washed three times with demineralized water, and then stained with an ALP 
solution/alizarin red solution for $10 \mathrm{~min}$. Finally, cells were washed three times with PBS.

ALP precipitates were solubilized to quantify ALP activity. Briefly, stained samples were incubated with $800 \mathrm{~mL}$ acetic acid (10\%) for $30 \mathrm{~min}$, the supernatant was transferred into a $1.5 \mathrm{~mL}$ tube and boiled for 10 $\min$ at $85^{\circ} \mathrm{C}$, and then the supernatant was placed on ice for $5 \mathrm{~min}$. After centrifugation (15,000/g, $15 \mathrm{~min}), 500$ $\mu \mathrm{L}$ of the supernatant was transferred into another 1.5$\mathrm{mL}$ tube and mixed with $200 \mu \mathrm{L}$ of $10 \%$ ammonium hydroxide. Samples were transferred into a 96-well microtiter plate and optical density was measured at 405 nm using a standard ELISA reader. $P$ was calculated with a student's $t$-test $(n=3)$ to detect statistically relevant differences.

\subsection{MSC-derived adipocytes}

Adipogenic culture of MSCs as reported (17) was performed to induce differentiation into adipocytes. Briefly, MSCs in the second or third passage were induced to form adipocytes in an adipogenic medium (a-MEM and 10\% FBS) for up to 12 days as determined by peak adipogenesis. At that point, the medium was changed to an adipocyte-sustaining medium consisting of high-glucose DMEM with $10 \mu \mathrm{g} / \mathrm{mL}$ insulin and $10 \%$ FBS to promote adipocyte maturity. Cultures were analyzed prior to adipogenesis on day 0 and at specific points in time over a period of 25 days.

\subsection{Oil Red O staining}

Cells were fixed with $4 \%$ paraformaldehyde for $20 \mathrm{~min}$ at $4^{\circ} \mathrm{C}$. Cells were rinsed and washed and then stained with Oil Red O solution for 15 min to stain lipid droplets/ vacuoles. Cells in random fields were manually counted and the number in 5 high power fields was averaged.

\subsection{Real-time PCR}

RNA treated with DNase was isolated from MSC-derived osteoblasts and adipocytes, enriched adipofibroblasts, and lipid-laden adipocyte cultures at specific timepoints using an RNeasy Mini Kit in accordance with the manufacturer's instructions (Qiagen). Cells were lysed on plates in buffer containing GITC (Buffer RLT). Reverse Transcription was performed immediately after RNA isolation using a Transcriptor First Strand cDNA synthesis kit and oligo-dT primers (Roche, Branchburg, NJ). Real-time PCR was performed using SybrGreen and Taqman technology. Briefly, $10 \mathrm{~mL}$ of SybrGreen Master Mix (Applied Biosystems, Darmstadt, Germany) was mixed with $1 \mathrm{~mL}(10 \mathrm{pg})$ of forward primer, $1 \mathrm{~mL}(10$ $\mathrm{pg})$ of reverse primer, $6.8 \mathrm{~mL}$ of water, and $1.2 \mathrm{~mL}(60$ $\mathrm{ng}$ ) of template. Levels of expression of the beta-actin gene were used to normalize mRNA expression for realtime PCR. Reactions were performed using the following conditions: $95^{\circ} \mathrm{C}$ for $10 \mathrm{~min}$ and cycles of $95^{\circ} \mathrm{C}$ for $15 \mathrm{sec}, 55-60^{\circ} \mathrm{C}$ for $30 \mathrm{sec}$, and $72^{\circ} \mathrm{C}$ for $30 \mathrm{sec}$ for 40 cycles. The primers used for each gene were as follows: Runx2: Forward GACAGTCCCAACTTCCTGTG, Reverse GCGGAGTAGTTCTCATCATTC; Osterix: Forward GCTCGTAGATTTCTATCCTC, Reverse CTTAGTGACTGCCTAACAGA; Collagen1: Forward TGACTGGAAGA GCGGA GA GTA, Reverse GACGGCTGAGTAGGGAACAC; Osteocalcin: Forward TGCCTGGCTGGAGATTCTG, Reverse GCT GCT G T GA CAT C CATA C T T; PPAR $\gamma$ : Forward GGAATTAGATGACAGTGACTTGGC, Reverse ATCTTCTGGAGCACCTTGGC; $\beta$-actin: Forward CCTCTATGCCAACACAGT, Reverse AGCCACCAATCCACACAG.

\subsection{Statistical analysis}

All values are expressed as the mean \pm SEM. Data were analyzed with the aid of SPSS database, and variance was evaluated with one-way ANOVA. Differences were considered significant at $p<0.05$.

\section{Results}

\subsection{The effects of DHEA on bone morphology}

Bone volume (BV), bone mineral density (BMD), and the number of trabecules in bone (Tb.N) were lower in the OVX group than in the sham-operated group ( $p$ $<0.05)$. Trabecular spacing (Tb.sp) was wider in the OVX group than in the sham-operated group $(p<0.05)$, suggesting that a mouse model of PMO was successfully established. Compared to the OVX group that was treated with saline, the OVX + DHEA and OVX + E2 groups had a higher BV, BMD, and Tb.N and a smaller Tb.sp, suggesting that DHEA and E2 improved bone morphology in mice with osteoporosis (Figure 1).

\subsection{DHEA increases osteoblastogenesis by up-regulating} the expression of osteoblastogenesis-related genes

In osteoblastogenic medium, DHEA promoted MSCs to differentiate into osteoblasts. The ALP activity of osteoblasts was higher in groups treated with DHEA or E2 than that in the control group $(p<0.05)$ (Figure $2 \mathrm{~A})$. The number of bone nodules also increased in the groups treated with DHEA or E2 compared to the control group $(p<0.05)$ (Figures $2 \mathrm{~B}$ and $2 \mathrm{C})$. The expression of mRNA by osteogenesis-related genes was examined using real-time PCR in order to explore the mechanisms by which DHEA regulates MSC differentiation. Compared to the control treatment, DHEA and E2 promoted expression of collagen I, osteocalcin, Runx2, and osterix. However, there was no difference in levels of expression in the two groups (Figure 2D). 

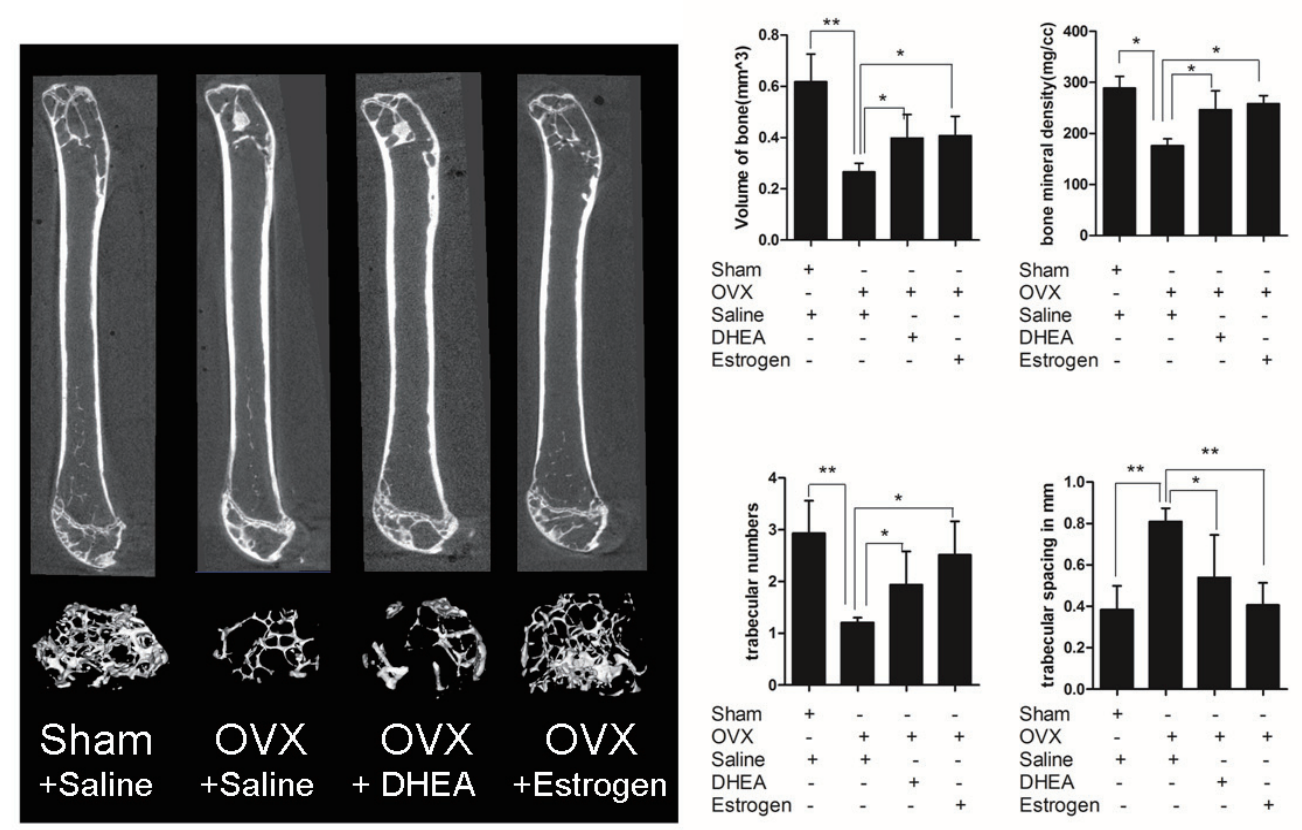

Figure 1. Effects of DHEA on bone morphology in OVX mice. Sham mice underwent mock surgery and received saline. Ovariectomized (OVX) mice underwent bilateral oophorectomy and were randomly divided into three groups: OVX (treated with saline), OVX + DHEA (treated daily with $5 \mathrm{~mL}$ of mixed raw herbs [BSNXD] per kg body weight), and OVX + E2 (treated daily with $5 \mathrm{~mL}$ of DHEA per kg body weight). Femur samples were harvested after 12 weeks of treatment. Micro-CT was performed to determine the bone morphology in femurs. Bone volume, bone mineral density, the number of trabecules in bone, and trabecular spacing were measured at the original magnification $(200 \times)$. Data are presented as the mean \pm SEM. $(n=6) .{ }^{*} p<$ $0.05,{ }^{* *} p<0.01$ compared to the OVX group.

A

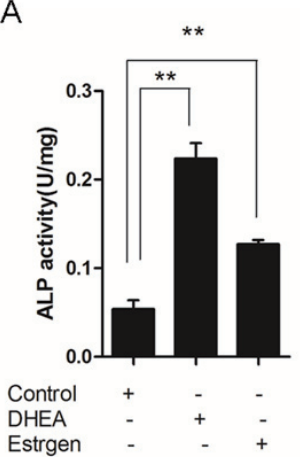

B
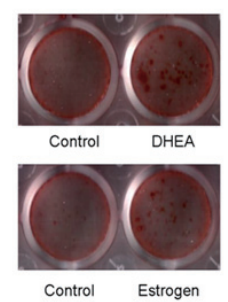

C

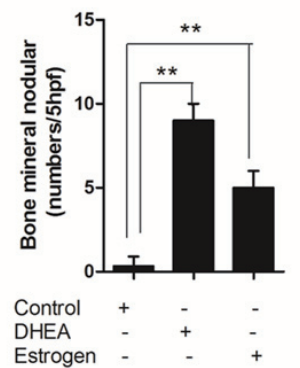

$\mathrm{D}$

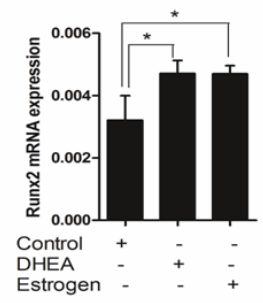

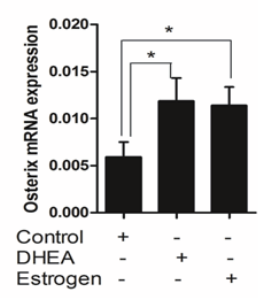

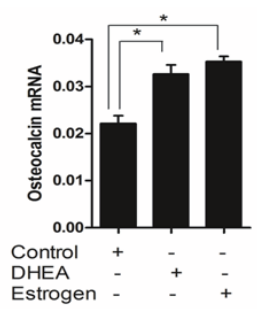

Control

DHEA
Estrogen

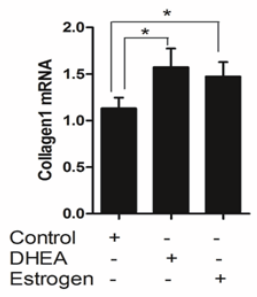

DHEA

Figure 2. DHEA increased ALP activity and expression of mRNA by osteoblastogenesis-related genes. Primary MSCs were exposed to control serum, $10^{-7} \mathrm{M}$ DHEA, or $10^{-9} \mathrm{M}$ E2 for $48 \mathrm{~h}$ under osteoblastogenic conditions. The ALP activity of osteoblasts was determined with a kit to analyze ALP activity after 7 days of culturing (2A), alizarin red staining was performed after 21 days of culturing (2B), and the number of mineralized bone nodules was counted (2C). Runx2, osterix, collagen I, and osteocalcin mRNA were analyzed (2D). Data are presented as the mean \pm SEM. $(n=6) .{ }^{*} p<0.05,{ }^{* *} p<0.01$ compared to the control group.

\subsection{DHEA increases the proportion of Foxp $3^{+}$Tregs in the spleen and enhances osteoblastogenesis}

Immune cells can influence bone metabolism, so this study determined whether immune cells were affected after DHEA treatment. Changes in spleen-derived immune cells after DHEA treatment were examined first. Compared to the sham-operated group, the OVX 

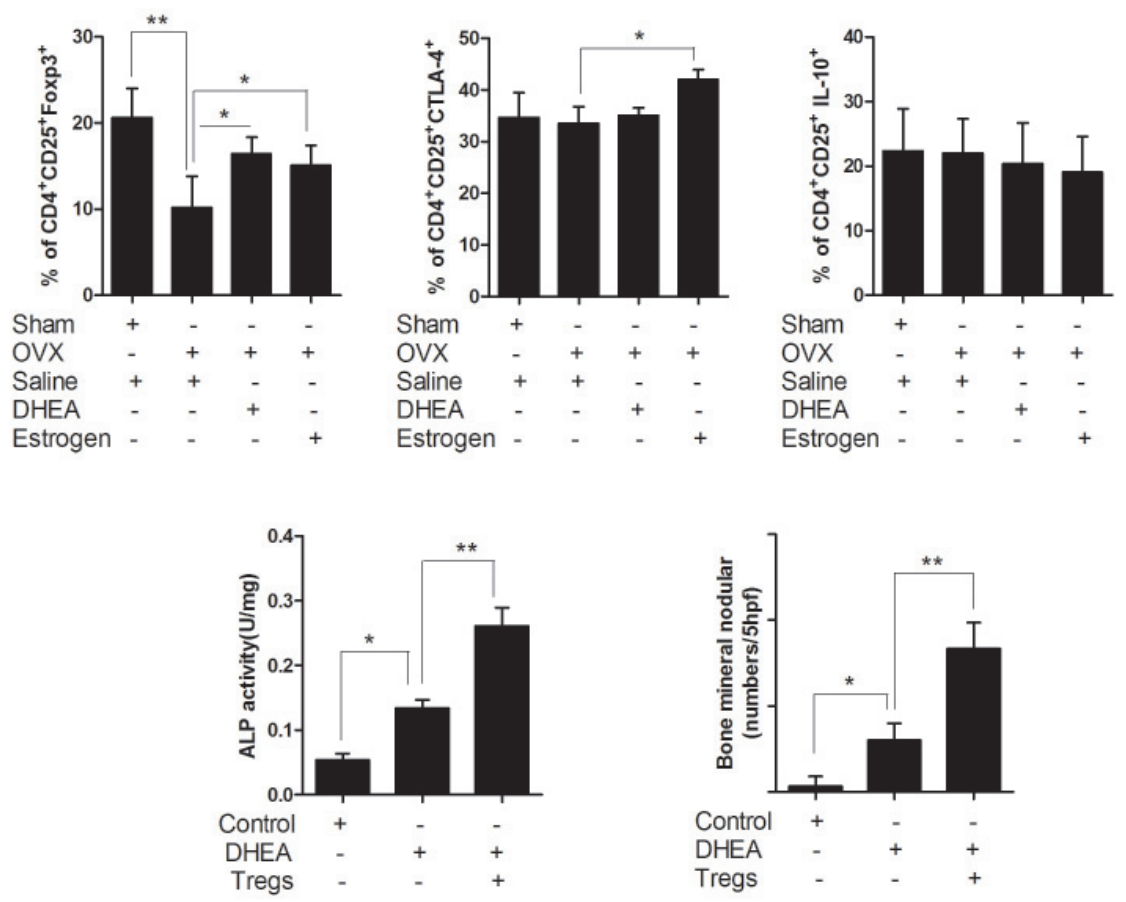

Figure 3. DHEA increased the number of Foxp3 ${ }^{+}$Tregs, and Tregs enhanced the effects of DHEA on osteoblast differentiation. Sham mice underwent mock surgery and received saline. OVX mice underwent bilateral oophorectomy and were randomly divided into three groups: OVX (treated with saline), OVX + DHEA (treated daily with $5 \mathrm{~mL}$ of E2 per kg body weight), and OVX + E2 (treated daily with $5 \mathrm{~mL}$ of E2 per kg body weight). Spleen samples were harvested after 12 weeks. Flow cytometry was performed to ascertain immune cells in the spleen. Data are presented as the mean $\pm \operatorname{SEM} .(n=6) .{ }^{*} p<0.05$, ${ }^{* *}$ $p<0.01$ compared to the OVX group. Primary MSCs were exposed to control serum, $10^{-7} \mathrm{M}$ DHEA, or $10^{-9} \mathrm{M} \mathrm{E} 2 \mathrm{for} 48 \mathrm{~h}$ under osteoblastogenic conditions in the presence or absence of Tregs. ALP activity of osteoblasts was determined using a kit to analyze ALP activity. The number of bone nodules was assessed using alizarin red staining. Data are presented as the mean \pm SEM. $(n=6)$. ${ }^{*} p<0.05,{ }^{* *} p<0.01$.

group had fewer Foxp3-positive Tregs. DHEA treatment increased the percentage of Foxp $3^{+}$Tregs $(p<0.05)$ while E2 did not. Conversely, E2 treatment increased the percentage of CTLA- $4^{+}$Tregs but DHEA did not significantly influence the number of Tregs (Figure $3)$. MSCs were cultured in the presence or absence of Tregs in order to determine the effects of Tregs on DHEA-mediated differentiation of MSCs. In the presence of Tregs, ALP activity was higher and bone nodular production increased, suggesting that Tregs can enhance the effect of DHEA on differentiation of MSCs (Figure 3).

\subsection{DHEA does not directly affect adipocyte differentiation}

MSCs can differentiate into osteoblasts and adipocytes. Increased numbers of adipocytes indicate a significant risk of PMO developing. MSCs cultured in osteoblastogenic medium and treated with DHEA had increased numbers of adipocytes. However, MSCs cultured in adipogenic medium and treated with DHEA or E2 did not produce different numbers of adipocytes. The transcription factor PPAR $\gamma$ regulates adipogenesis. Expression of this gene was assessed using real-time PCR, and the levels of expression in the group treated with DHEA did not differ from those in the control group and the group treated with E2 (Figure 4).

\section{Discussion}

Mature OVX mice were previously used to model postmenopausal bone loss (18). Analysis of bone morphology has revealed that OVX mice have significantly reduced bone mass, bone density, and fewer trabecules in bone as well as significantly wider trabecular spacing compared to sham-operated mice (19). These findings were evident in the present study, suggesting that a model of PMO was successfully established.

After treatment with DHEA, the OVX mice had significantly increased bone mass, bone density, and more trabecules in bone as well as significantly smaller trabecular spacing, suggesting that DHEA significantly improves bone morphology. An improvement in bone morphology can increase the mechanical strength and integrity of bone and affect bone growth, suggesting that DHEA has clinical significance in the prevention and treatment of PMO.

Runx 2 and osterix are transcription factors associated with osteoblastogenesis while collagen 1 and osteocalcin are genes expressed in osteoblasts; all four of these genes are markers of different stages of bone 

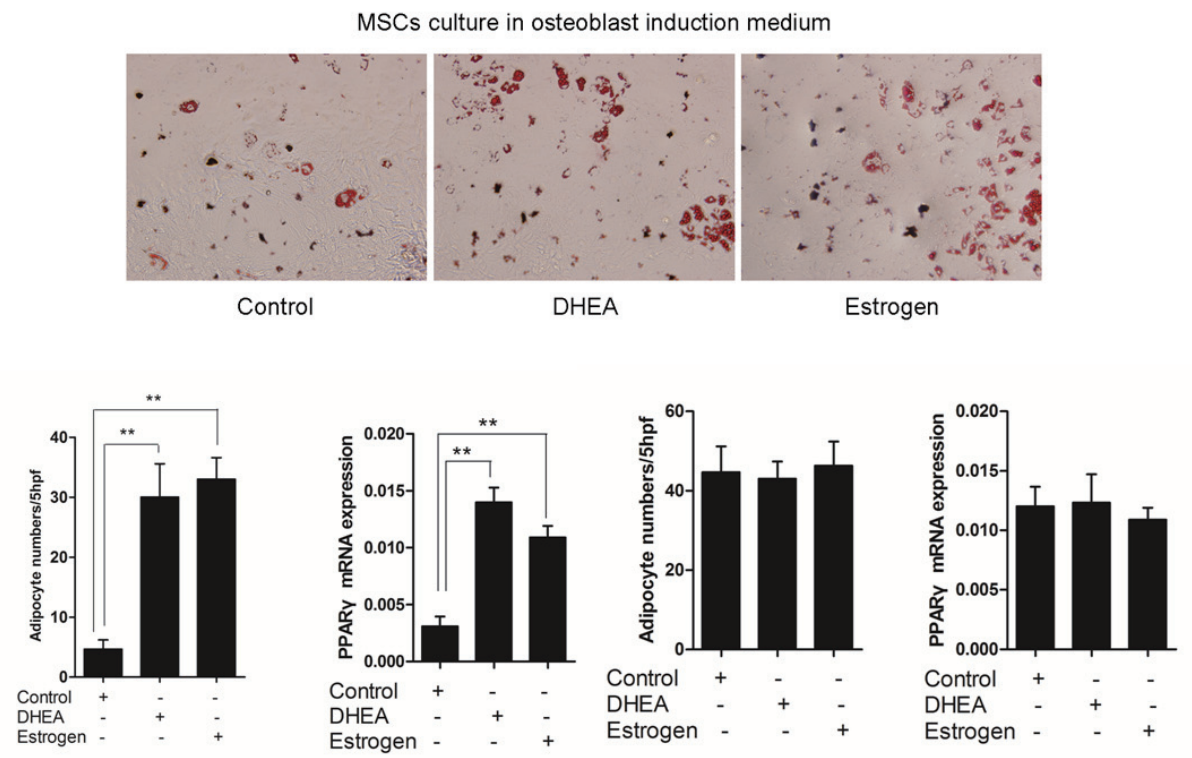

Figure 4. DHEA affected adipocyte differentiation via osteoblast production. Primary MSCs were exposed to control serum, $10^{-7} \mathrm{M}$ DHEA, or $10^{-9} \mathrm{M}$ E2 for $48 \mathrm{~h}$ under adipogenic conditions. The number of adipocytes was assessed using oil red $\mathrm{O}$ staining. Expression of PPAR $\gamma$ mRNA was assessed using real-time PCR. Data are presented as the mean \pm SEM. $(n=6) .{ }^{*} p<0.05$, ${ }_{* *} p<0.01$ compared to the OVX group.

development (20-22). PPAR $\gamma$ is a transcriptional factor that regulates adipogenesis (23). Although DHEA increases the number of adipocytes in osteoblastogenic medium, it produces no changes in the number of adipocytes in adipogenic medium (24-30).

Tregs affect bone metabolism, and this action is directly regulated by Foxp3 and CTLA-4 or it is indirectly regulated by TGF- $\beta$ and IL-10 (31-36). DHEA can markedly increase the proportion of Foxp $3^{+}$ Tregs and encourage osteoblast differentiation to prevent PMO. DHEA does not affect the ratio of CTLA $4^{+}$or IL $-10^{+}$Tregs, suggesting that DHEA plays a role on osteoblast differentiation via Foxp3.

In a previous study, the current authors found that DHEA significantly promoted proliferation and inhibited apoptosis of osteoblast via mitogen-activated protein kinase signaling pathways independent of either androgen receptors or estrogen receptors, suggesting that it may play a direct role via a DHEA-specific receptor instead of via conversion to androgens or estrogens ( 6 ).

Results of in vivo and ex vivo experiments revealed that DHEA can directly promote differentiation of MSCs into osteoblasts. DHEA increased the expression of Runx2, Collagen1, Osterix, and Osteocalcin mRNA, increasing the ALP activity of osteoblasts and the number of mineralized bone nodules. DHEA increases the number of Foxp $3^{+}$ Tregs, increasing osteoblast differentiation. Therefore, DHEA is likely to be an ideal solution to prevent and treat PMO.

\section{Acknowledgements}

This work was supported by a grant from the National Natural Science Foundation of China (grant no. 81401171 to XM Qiu), a grant from the National Natural Science Foundation of China (grant no. 31571196 to L Wang), a grant from the Shanghai Municipal Science and Technology Commission for a 2015 Science and Technology Project to Guide Medicine (project no. 15401932200; L Wang), a grant from the 2008 JSPS Postdoctoral Fellowship for Foreign Researchers (P08471; L Wang), a grant from the National Natural Science Foundation of China (grant no. 30801502; L Wang), a grant from the Shanghai Pujiang Program (number 11PJ1401900; L Wang), a grant from the Program for Outstanding Leaders in Medicine (D-J Li), and a grant from the Shanghai Project to Develop Key Disciplines in Medicine (Integrated Chinese and Western Medicine).

\section{References}

1. Wang L, Hao Q, Wang YD, Wang WJ, Li DJ. Protective effects of dehydroepiandrosterone on atherosclerosis in ovariectomized rabbits via alleviating inflammatory injury in endothelial cells. Atherosclerosis. 2011; 214:4757.

2. El Maghraoui A, Rezqi A, El Mrahi S, Sadni S, Ghozlani I, Mounach A. Osteoporosis, vertebral fractures and metabolic syndrome in postmenopausal women. BMC Endocr Disord. 2014; 14:93.

3. Caputo EL, Costa MZ. Influence of physical activity 
on quality of life in postmenopausal women with osteoporosis. Rev Bras Reumatol. 2014; 54:467-473.

4. Hoshiba T, Kawazoe N, Chen G. The balance of osteogenic and adipogenic differentiation in human mesenchymal stem cells by matrices that mimic stepwise tissue development. Biomaterials. 2012; 33:2025-2031.

5. Hwang NS, Varghese S, Elisseeff J. Controlled differentiation of stem cells. Adv Drug Deliv Rev. 2008; 60:199-214

6. Wang L, Wang YD, Wang WJ, Li DJ. Differential regulation of dehydroepiandrosterone and estrogen on bone and uterus in ovariectomized mice. Osteoporos Int. 2009; 20:79-92.

7. Wang L, Wang YD, Wang WJ, Zhu Y, Li DJ. Dehydroepiandrosterone improves murine osteoblast growth and bone tissue morphometry via mitogenactivated protein kinase signaling pathway independent of either androgen receptor or estrogen receptor. J Mol Endocrinol. 2007; 38:467-79.

8. Wang Y D, Wang L, Li D J, Wang W J . Dehydroepiandrosterone inhibited the bone resorption through the upregulation of OPG/RANKL. Cell Mol Immunol. 2006; 3:41-46.

9. Faienza MF, Ventura A, Marzano F, Cavallo L. Postmenopausal osteoporosis: The role of immune system cells. Clin Dev Immunol. 2013; 2013:575936.

10. Danks L, Takayanagi H. Immunology and bone. J Biochem. 2013; 154:29-39.

11. Dziak R. Osteoimmunology: Cross-talk between bone and immune cells. Immunol Invest. 2013; 42:657-660.

12. Yin $\mathrm{G}$, Wen $\mathrm{FQ}$. Immune mediated inflammatory pathogenesis and assessment of disease activity in rheumatoid arthritis. Sichuan Da Xue Xue Bao Yi Xue Ban. 2015; 46:267-271.

13. Yuan FL, Li X, Lu WG, Xu RS, Zhao YQ, Li CW, Li JP, Chen FH. Regulatory T cells as a potent target for controlling bone loss. Biochem Biophys Res Commun. 2010; 402:173-176.

14. Cuijpers VM, Alghamdi HS, Van Dijk NW, Jaroszewicz J, Walboomers XF, Jansen JA. Osteogenesis around CaPcoated titanium implants visualized using 3D histology and micro-computed tomography. J Biomed Mater Res A. 2015; 103:3463-3473.

15. Costa-Pinto AR, Correlo VM, Sol PC, Bhattacharya M, Charbord P, Delorme B, Reis RL, Neves NM. Osteogenic differentiation of human bone marrow mesenchymal stem cells seeded on melt based chitosan scaffolds for bone tissue engineering applications. Biomacromolecules. 2009; 10:2067-2073.

16. Vater C, Kasten P, Stiehler M. Culture media for the differentiation of mesenchymal stromal cells. Acta Biomater. 2011; 7:463-477.

17. Nuttall ME, Gimble JM. Controlling the balance between osteoblastogenesis and adipogenesis and the consequent therapeutic implications. Curr Opin Pharmacol. 2004; 4:290-294

18. Hempel E, Günther P, Neumann G, Müller B. Experimental studies on the castration osteoporosis in the rat. II. Behavior of bone minerals. Zentralbl Gynakol. 1978; 100:1391-1398.

19. Tuomikoski P, Ylikorkala O, Mikkola TS. Postmenopausal hot flushes and bone mineral density: A longitudinal study. Acta Obstet Gynecol Scand. 2015; 2:198-203.

20. Kahler RA, Yingst SM, Hoeppner LH, Jensen ED, Krawczak D, Oxford JT, Westendorf JJ. Collagen 11a1 is indirectly activated by lymphocyte enhancer-binding factor 1 (Lef1) and negatively regulates osteoblast maturation. Matrix Biol. 2008; 27:330-338.

21. Lu X, Gilbert L, He X, Rubin J, Nanes MS. Transcriptional regulation of the osterix (Osx, Sp7) promoter by tumor necrosis factor identifies disparate effects of mitogenactivated protein kinase and NF kappa B pathways. J Biol Chem. 2006; 281:6297-6306.

22. Viereck V, Siggelkow H, Tauber S, Raddatz D, Schutze N, Hüfner M. Differential regulation of Cbfa1/Runx2 and osteocalcin gene expression by vitamin-D3, dexamethasone, and local growth factors in primary human osteoblasts. J Cell Biochem. 2002; 86:348-356.

23. Duque G. Will reducing adopogenesis in bone increase bone mass?: PPARgamma2 as a key target in the treatment of age-related bone loss. Drug News Perspect. 2003; 16:341-346.

24. Cao JJ. Effects of obesity on bone metabolism. J Orthop Surg Res. 2011; 6:30.

25. Colaianni G, Brunetti G, Faienza MF, Colucci S, Grano M. Osteoporosis and obesity: Role of Wnt pathway in human and murine models. World J Orthop. 2014; 5:242246.

26. Rosen CJ, Bouxsein ML. Mechanisms of disease: Is osteoporosis the obesity of bone? Nat Clin Pract Rheumatol. 2006; 2:35-43.

27. Foresta C, Strapazzon G, De Toni L, Gianesello L, Calcagno A, Pilon C, Plebani M, Vettor R. Evidence for osteocalcin production by adipose tissue and its role in human metabolism. J Clin Endocrinol Metab. 2010; 95:3502-3506.

28. Maurin AC, Chavassieux PM, Frappart L, Delmas PD, Serre CM, Meunier PJ. Influence of mature adipocytes on osteoblast proliferation in human primary cocultures. Bone. 2000; 26:485-489.

29. Ali AT, Penny CB, Paiker JE, van Niekerk C, Smit A, Ferris WF, Crowther NJ. Alkaline phosphatase is involved in the control of adipogenesis in the murine preadipocyte cell line, 3T3-L1. Clin Chim Acta. 2005; 354:101-109.

30. Ikeda K, Takeshita S. Factors and mechanisms involved in the coupling from bone resorption to formation: How osteoclasts talk to osteoblasts. J Bone Metab. 2014; 21:163-167.

31. Zhang Y, Khan D, Delling J, Tobiasch E. Mechanisms underlying the osteo- and adipo-differentiation of human mesenchymal stem cells. Scientific World Journal. 2012; 2012:793823.

32. Luo C, Wang L, Sun C, Li Dj. Estrogen enhances the functions of $\mathrm{CD} 4^{+} \mathrm{CD} 25^{+} \mathrm{Foxp}^{+}$regulatory $\mathrm{T}$ cells that suppress osteoclast differentiation and bone resorption in vitro. Cell Mol Immunol. 2010; 1:50-8.

33. Churlaud G, Pitoiset F, Jebbawi F, Lorenzon R, Bellier B, Rosenzwajg M, Klatzmann D. Human and mouse $\mathrm{CD} 8^{+} \mathrm{CD} 25^{+} \mathrm{FOXP} 3^{+}$regulatory $\mathrm{T}$ cells at steady state and during interleukin-2 therapy. Front Immunol. 2015; 6:171.

34. Ligeiro de Oliveira AP, Peron JP, Santos Franco AL, Golega BA, Paula Vieira R, Ibanez OC, Ribeiro OG, Cabrera WH, De Franco M, Oliveira-Filho RM, Rizzo LV, Vargaftig BB, de Lima WT. Ovariectomized OVAsensitized mice display increased frequency of $\mathrm{CD} 4^{+}$ Foxp $3^{+} \mathrm{T}$ regulatory cells in the periphery. PLoS One. 2013; 8:e65674.

35. Liu JC, Zhou CH, Zhang X, Chen Y, Xu BL, Cui L, Xu DH. Effect of 1,25-dihydroxyvitamin D-3 on regulatory 
T cells in ovariectomized mice. Biomedical and Environmental Sciences. 2014; 27:779-785.

36. Zaiss MM, Frey B, Hess A, Zwerina J, Luther J, Nimmerjahn F, Engelke K, Kollias G, Hünig T, Schett G, David JP. Regulatory T cells protect from local and systemic bone destruction in arthritis. J Immunol. 2010; 184:7238-7246.

(Received May 30, 2015; Revised September 9, 2015; Accepted October 7, 2015) 